\section{Decompression side tube-equipped double-balloon enteroscopy extends intubation depth and reduces patient discomfort}

Since the first article introducing doubleballoon endoscopy (DBE) was published in 2001 [1], the technique has proven effective for deep intubation of the small bowel and is used in clinical practice worldwide. However, the intubation
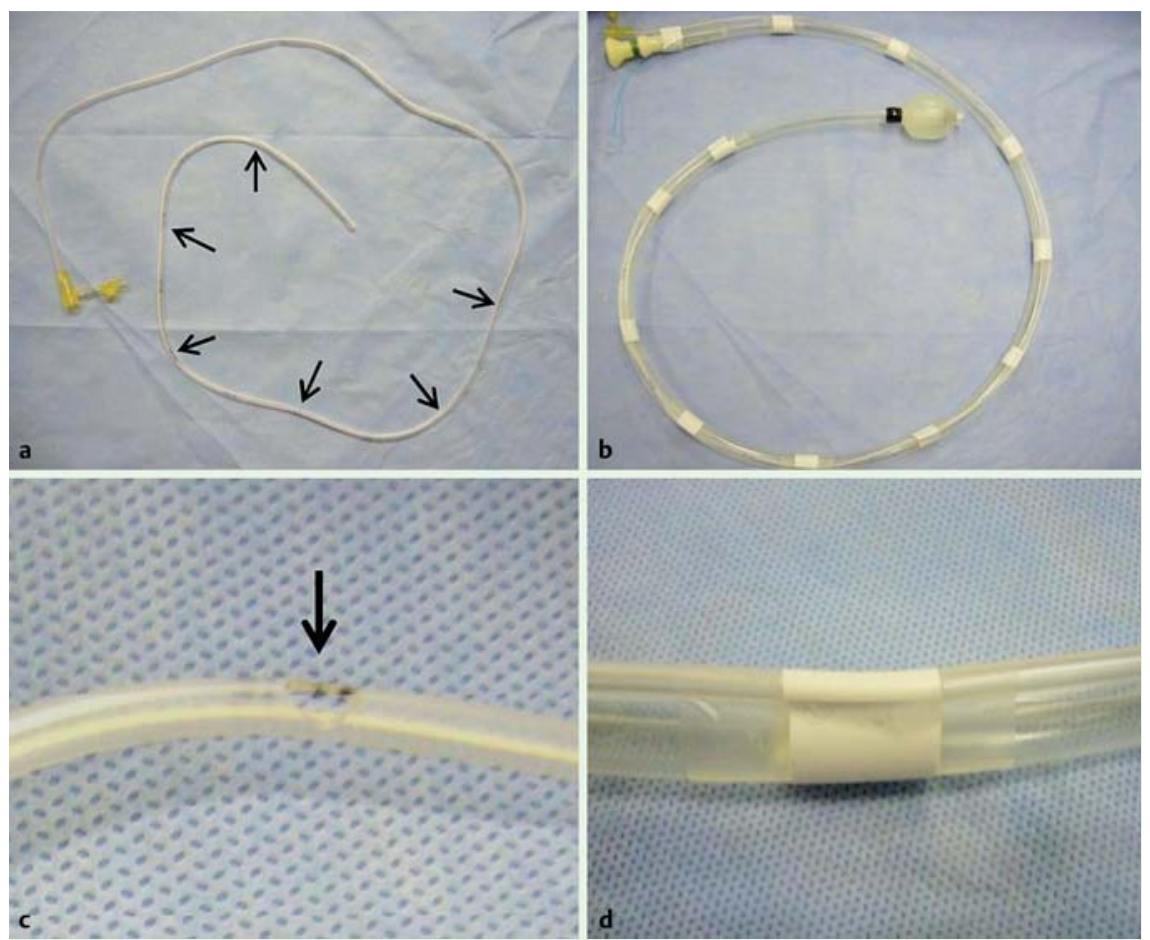

depth is sometimes limited by the distension of the small bowel due to air insufflation during the procedure. In a recent report [2], the total success rate using a combined (antegrade and retrograde) or antegrade-only approach in DBE was

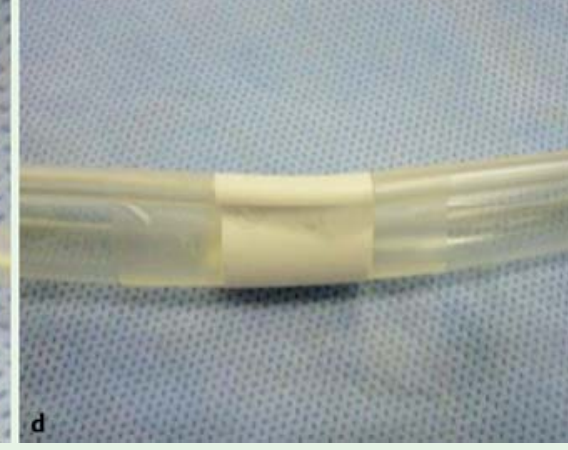

Fig. 1 Decompression side tube attached to the DBE overtube. a A nasogastric tube with sideholes placed every $10 \mathrm{~cm}$ (arrows) is used as the decompression side tube. $\mathbf{b}$ The decompression tube and the overtube are attached to each other after aligning the proximal ends. $c$ Sidehole in the nasogastric tube (arrow). $\mathbf{d}$ Point of attachment with tape.

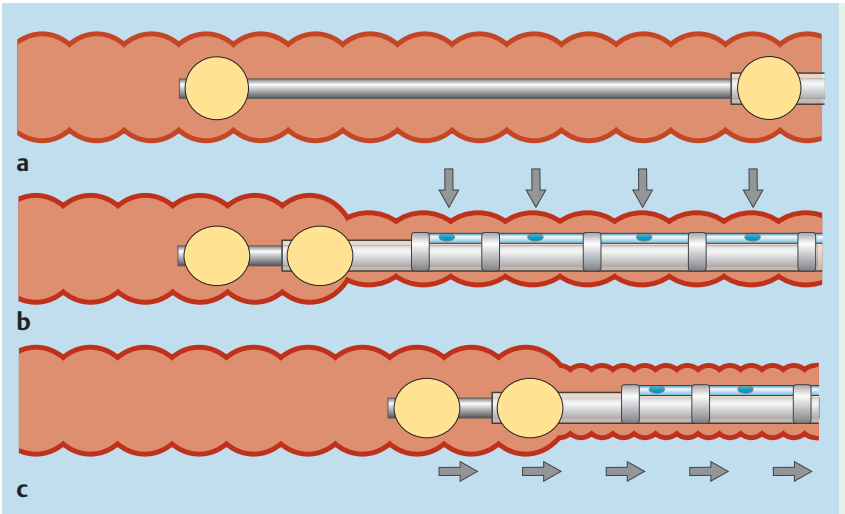

Fig.2 Schematic diagram of decompression side tube-equipped double-balloon enteroscopy (DBE). $\mathbf{a}$ The DBE device is inserted into the small intestine with air insufflation. $\mathbf{b}$ Intestinal gas and fluid around the enteroscope are spontaneously drained through the decompression side tube. c Intraluminal decompression leads to effective shortening of the proximal small intestine.
$44.0 \%$ (95\% confidence interval [CI] $35.0 \%-53.3 \%)$. To improve intubation depth, we have developed a modified method for desufflation around the overtube of DBE using a decompression side tube.

A nasogastric tube with sideholes placed every $10 \mathrm{~cm}$ ( Fig. 1 a) is used as the decompression side tube. The proximal ends of this tube and the overtube are aligned, and the two tubes are attached to each at several points using plastic tape and film dressing tape ( Fig. $1 \mathbf{b}-\mathbf{d}$ ). Decompression tube-equipped DBE is carried out with air insufflation. In this method, intestinal gas and fluid spontaneously drain out through the decompression tube ( Fig.2a,b). If necessary, manual aspiration is also possible. Intraluminal decompression leads to efficient insertion of the endoscope and shortening of the proximal small intestine ( $\bullet$ Fig. 2 c). We have not encountered any significant complications such as intraluminal mucosal injury with this method.

Recently, usefulness of a carbon dioxide $\left(\mathrm{CO}_{2}\right)$ insufflation system during DBE has also been reported [3]. However, decompression side tube-equipped DBE extends intubation depth and reduces patient discomfort associated with over-insufflation, even in cases where $\mathrm{CO}_{2}$ insufflation cannot be used. Total enteroscopy was successful using the antegrade-only approach and the EN-450T5 double-balloon enteroscope (Fujinon, Omiya, Japan) equipped with a decompression side tube ( Fig.3). Because this method is simple,

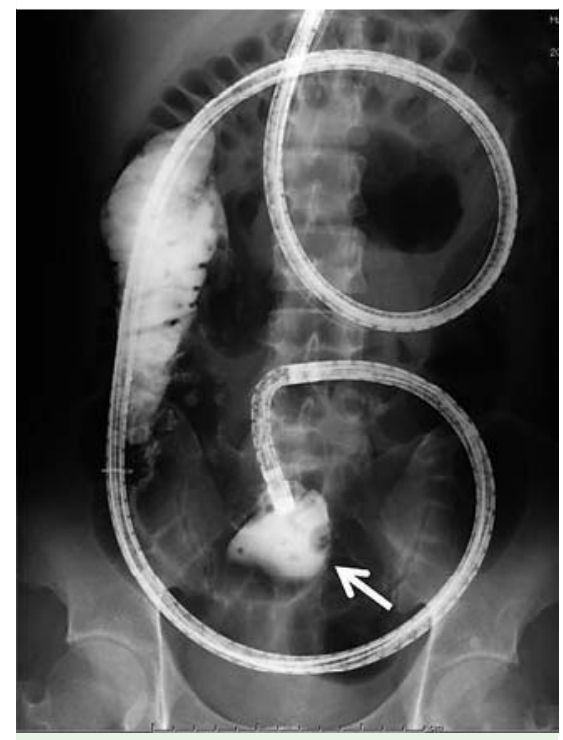

Fig. 3 X-ray image of decompression side tube-equipped double-balloon enteroscopy (EN-450T5 model) inserted from the mouth to the cecum (arrow). 
easily applied, and cost-effective, it may lead to a higher diagnostic and therapeutic yield via enteroscopy, while reducing patient discomfort.

Endoscopy_UCTN_Code_TTT_1AP_2AD

Competing interests: None

\section{K. Ikeya',2, S. Osawa', S. Kuriyama', Y. Hamaya ${ }^{1}$, T. Yamada ${ }^{1}$, M. Sugimoto ${ }^{1}$, T. Furuta ${ }^{3}$, K. Sugimoto ${ }^{1}$}

${ }^{1}$ First Department of Medicine, Hamamatsu University School of Medicine, Hamamatsu, Japan

${ }^{2}$ Center for Gastroenterology and Inflammatory Bowel Disease Research, Hamamatsu South Hospital, Hamamatsu, Japan

${ }^{3}$ Center for Clinical Research, Hamamatsu University School of Medicine, Hamamatsu, Japan

\section{References}

1 Yamamoto $H$, Sekine $Y$, Sato $Y$ et al. Total enteroscopy with a nonsurgical steerable double-balloon method. Gastrointest Endosc 2001; 53: 216-220

2 Xin L, Liao Z, Jiang YP et al. Indications, detectability, positive findings, total enteroscopy, and complications of diagnostic double-balloon endoscopy: a systematic review of data over the first decade of use. Gastrointest Endosc 2011; 74: 563-570

3 Bretthauer M, Lynge AB, Thiis-Evensen $E$ et al. Carbon dioxide insufflation in colonoscopy: safe and effectiveness in sedated patients. Endoscopy 2005; 37: 706 - 709

\section{Bibliography}

Dol http://dx.doi.org/ 10.1055/s-0032-1309356 Endoscopy 2012; 44: E256-E257

(c) Georg Thieme Verlag KG

Stuttgart · New York

ISSN 0013-726X

\section{Corresponding author}

S. Osawa, MD, PhD, Senior Assistant Professor

First Department of Medicine

Hamamatsu University School of Medicine

1-20-1 Handayama

Higashi-ku

Hamamatsu 431-3192

Japan

Fax: +81-53-434-9447

sososawa@hama-med.ac.jp 